\title{
Modelo de apoio à decisão entre produzir ou terceirizar
}

\section{Decision support model to make or outsource}

João Paulo Santos Aragão ${ }^{1}$ Cínthia Ladjane de Souza Holanda ${ }^{2}$ Marcele Elisa Fontana ${ }^{3}$

${ }^{1}$ Universidade Federal de Pernambuco (UFPE), Campus do Agreste (CAA), Caruaru (PE), Brasil. Mestrando no Programa de Pós-Graduação em Engenharia de Produção do CAA (PPGEP-CAA) aragaopaulojoao@gmail.com

${ }^{2}$ Faculdade de Belo Jardim (FBJ), Belo Jardim (PE), Brasil. Mestre em Engenharia de Produção (PPGEP-CAA) cinthia_holanda@hotmail.com

${ }^{3}$ Universidade Federal de Pernambuco (UFPE), Campus do Agreste (CAA), Caruaru (PE), Brasil. Doutor em Engenharia de Produção, docente permanente PPGEPCAA e líder do grupo de pesquisa em Decisões Estratégicas em Produção e Logística (DEPLog) marcele.elisa@ufpe.br

\begin{abstract}
Resumo
A decisão entre produzir ou terceirizar envolve múltiplos critérios, como custos, qualidade, aspectos gerenciais, entre outros, muitas vezes conflitantes entre si. A fim de evitar erros clássicos cometidos, em que geralmente considera-se apenas o custo como critério de avaliação, faz-se necessário determinar os critérios relevantes. Neste sentido, a utilização de uma abordagem formal para apoio à decisão torna-se fundamental para que o decisor adote a melhor alternativa, considerando as suas preferências e o conjunto de critérios envolvidos. Desta forma, este trabalho propôs um modelo de apoio à decisão entre produzir ou terceirizar, por meio do uso de Mapa Cognitivo e de um método multicritério de apoio à decisão (MCDA). Um estudo de caso no Arranjo Produtivo Local (APL) de confecções do Agreste Pernambucano, Brasil, foi apresentado. O modelo proposto foi capaz de auxiliar na definição dos critérios de avaliação, apoiando a tomada decisão de forma eficaz.
\end{abstract}

Palavras-chave: Terceirização. Mapa Cognitivo. Método PROMETHEE II.

\section{Abstract}

Make or buy decision involves multiple criteria as cost, quality, managerial aspects, among others, often conflicting with each other. In order to avoid classical mistakes, where it is generally considered only cost as an evaluation criterion, it is necessary to determine the relevant criteria. In this sense, the use of a formal approach to support decision making is essential for the decision maker to adopt the best alternative, considering their preferences and the set of criteria involved. Thus, this work proposed a decision support model between make or buy through the use of Cognitive Map and a multicriteria decision analysis (MCDA) method. A case study in the Local Productive Arrangement (APL) of clothing in Pernambuco, Brazil, was presented. The proposed model was able to assist in the definition of the evaluation criteria, supporting the decision making effectively.

Keywords: Outsourcing. Cognitive Map. PROMETHEE II method. 


\section{Introdução}

No ambiente empresarial cada vez mais competitivo e global, a terceirização é usada pelas empresas como uma estratégia de gestão e opção de modernização, posicionamento competitivo e como adequação às necessidades do mercado (Valença \& Barbosa, 2002). Além disso, vem sendo utilizada na execução de objetivos de desempenhos competitivos, como flexibilidade, capacidade de resposta, preço, qualidade e credibilidade, o que possibilita a empresa focar em suas competências essenciais (core competences) e procurar parcerias para complementar seus objetivos estratégicos (Gunasekaran, Irani, Choy, Filippi, \& Papadopoulos, 2015; Cervi \& Carpinetii, 2017).

No Brasil, o setor de prestação de serviços de terceirização vem exercendo influências significativas na economia e nas relações de trabalho das empresas. Essa influência favoreceu a abertura da economia brasileira para o mercado externo e contribuiu para a expansão de diversos setores produtivos no país (Departamento Intersindical de Estatística e Estudos Socioeconômicos [DIEESE], 2012). Portanto, entre os adeptos da terceirização destacam-se as empresas que compõem o setor da indústria têxtil e de confecção (Associação Brasileira da Indústria Têxtil e de Confecção [ABIT], 2013).

Frente a esse cenário destaca-se o Estado de Pernambuco. Terceiro maior Estado do Brasil no setor de confecções, evidenciam-se no Estado Pernambucano os polos de confecções da Região Metropolitana do Recife e da Região Agreste, esta última ambiente de estudo deste trabalho (Associação Brasileira da Indústria Têxtil e de Confecção [ABIT], 2015). Como característica das atividades de confecção dessa região, observa-se a adoção da terceirização como uma das principais formas de flexibilização de trabalho.

Por conseguinte, de acordo com Piccinini, Oliveira e Fontoura (2006), a contratação de outras empresas para a realização de algumas etapas do processo produtivo é uma prática comum para atender a sazonalidade da produção, permitindo às empresas do segmento de confecção flexibilizar a quantidade de trabalhadores na produção, reduzir custos e dimensionar a capacidade produtiva de acordo com o volume de pedidos.

Além disso, ressalta-se que a maioria das empresas que constituem o Arranjo Produtivo Local (APL) de confecções do Agreste Pernambucano são empresas de médio e pequeno porte. Sendo assim, na perspectiva de Freitas, Schlindwein e Barth (2010), as pequenas e médias empresas (PME) requerem um maior conhecimento, informações de mercado e habilidades dos gestores e empresários para obtenção de êxito organizacional. Ainda segundo os autores, estas empresas encontram-se mais suscetíveis à competitividade e aos riscos de ordem legal, administrativa e financeira que a má decisão de terceirização pode acarretar, influenciando inclusive nas atividades centrais desempenhadas pela empresa e, consequentemente, no seu resultado econômico. 
Desta forma, quando as empresas terceirizam uma significativa parte de seu negócio, uma tomada de decisão equivocada pode trazer consequências negativas, tais como: custos excedentes, devido à escolha inadequada da empresa contratada; o não cumprimento de prazos; a falta de qualidade dos produtos ou serviços prestados; problemas relacionados a contratos legais; a perda de mercado e até mesmo falência da organização.

Outro fator que pode acarretar no insucesso da terceirização é quando as empresas consideram apenas a redução de custos como critério de decisão. Assim, a terceirização tem por objetivo principal não apenas a redução de custos, mas também trazer agilidade, flexibilidade e competitividade às empresas, sendo, portanto, uma decisão estratégica. Não obstante, ainda, assim, a terceirização é vista como uma decisão de curto prazo, muitas vezes baseadas nas experiências passadas dos gestores e empresários. Porém, por ser estratégica, a decisão de terceirização envolve múltiplos critérios que podem ser conflitantes entre si. Portanto, a formalização e estruturação de um modelo de decisão busca tornar a decisão sobre terceirização mais eficiente nas cadeias de suprimentos.

Em vista disso, objetivou-se neste trabalho propor um modelo que auxilie na tomada de decisão na escolha entre produzir ou terceirizar no APL de confecções do Agreste Pernambucano. O modelo proposto foi baseado no uso de Mapa Cognitivo e no método multicritério de apoio à decisão (MCDA), O PROMETHEE II.

Além desta introdução, o trabalho conta com outras cinco Secções. Na Secção 2 apontam-se os conceitos gerais sobre terceirização e os métodos utilizados para elaboração do modelo proposto. $\mathrm{Na}$ Secção 3 é apresentado o modelo de apoio à decisão. Na sequência, apresenta-se o estudo de caso. A Secção 5 discute os resultados alcançados. E, por fim, algumas considerações finais são feitas na Secção 6.

\section{Base conceitual \\ 2.1 Terceirização}

O que hoje se conhece por terceirização de atividades das organizações é tido como um fenômeno antigo, iniciando-se nos Estados Unidos com o advento da II Guerra Mundial (Ensslin, Giffhorn, Ensslin, \& Vianna, 2010). Desta forma, a terceirização é vista como a transferência de um conjunto de funções ou processos de uma organização para uma empresa externa ou até mesmo para um grupo de indivíduos (Kotabe \& Zhao, 2002; Kahraman, Engin, Kabak, \& Kaya, 2009).

Numa visão mais ampla, a terceirização se apoia, principalmente, no conceito de competência essencial (core competence) proposto por Prahalad e Hamel (1990). Sendo assim, possibilita a empresa focar em suas competências essenciais e procurar parcerias para complementar seus objetivos estratégicos (Gunasekaran, Irani, Choy, Filippi, \& Papadopoulos, 2015; Pereira, Scur, \& Consoni, 2017). 
Portanto, organizações antes verticalizadas foram obrigadas a concentrarem-se em suas competências essenciais e terceirizar as suas atividades de apoio (Oliveira, Contador, Shibao, \& Vendrametto, 2013). Por isso, a decisão sobre a terceirização demonstra ser uma decisão tipicamente estratégica.

Diversos são os motivos impulsionadores para terceirizar atividades, entre eles destacam-se: custos gerais mais baixos, por meio da força de trabalho menor; melhorar os processos por intermédio do beneficiamento de terceiros; melhorar foco nas atividades principais e; redução de riscos, por meio da transferência dos mesmos para os terceiros (Murthy, Karim, \& Ahmadi, 2015). Cachon e Harker (2002) consideraram que um dos principais motivos da busca da terceirização pelas empresas está relacionado ao custo de produção pela economia de escala. Além disso, para Xiao, Xia e Zhang (2007), a confiança e a qualidade oferecida pelos terceiros aos seus contratantes poderiam criar uma melhor percepção do consumidor sobre o produto destinado.

Naturalmente, a terceirização tem sido abordada aplicando uma série de teorias e métodos em diferentes contextos. Lonsdale (1999) e Fill e Visser (2000) propuseram modelos de decisão baseados na Teoria dos Custos de Transação, aplicados ao setor de tecnologia e elétrico, respectivamente. De forma mais ampla, Qureshi, Salman, e Khalid (2013) apresentaram um modelo baseado no conceito de competência essencial por intermédio da ferramenta Balanced Scorecard (BSC), aplicado em uma indústria de eletrônicos.

São encontrados na literatura trabalhos que utilizam a metodologia multicriterial para seleção de fornecedores (empresas terceirizadas) como, por exemplo: A.T. de Almeida (2007) que utilizou em serviços de transportes; Kahraman, Engin, Kabak e Kaya (2009) no setor moveleiro; e Peng (2012) no segmento alimentício.

Antes de aplicar um método multicritério é importante compreender os aspectos relacionados ao problema, bem como as suas influências e complexidades. Uma forma de melhor compreender o problema é por meio do uso de Mapas Cognitivos.

\subsection{Mapas Cognitivos}

Os mapas cognitivos $(\mathrm{MC})$ são tratados na literatura como uma ferramenta de auxílio à estruturação de problemas. A aplicação de métodos de estruturação de problemas permite uma melhor definição do problema, obtendo e capturando os elementos e as percepções dos diversos atores envolvidos no processo decisório, permitindo a geração de um maior conhecimento sobre o problema (Minaei, 2014).

Os MC têm como objetivo mapear o pensamento de uma pessoa sobre um problema ou assunto e são caracterizados por uma estrutura hierárquica, geralmente construídos na forma de um grafo, com 
meios e fins, além de um objetivo situado no topo da hierárquica, sendo suas estruturas ligadas por setas, as quais dão um sentido de causalidade, indicando que uma ideia pode conduzir a outra ou ter implicações nela (Eden, 2004). Ainda segundo Eden (2004), os MC podem ser usados para extrair modelos mentais de algum decisor, em que geralmente são obtidos por intermédio de entrevistas para a representação formal do mundo subjetivo do entrevistado.

Segundo Ensslin e Montibeller (1998), um mapa cognitivo pode ser definido como uma hierarquia de conceitos, dispostos a partir de ligações meios e fins. Trata-se da esquematização a partir da qual o decisor tornará explícito seu sistema de valores e poderá fornecer um conjunto de ações em potencial; processo este que será identificado a partir dos conceitos superiores e subordinados na hierarquia, respectivamente.

Na perspectiva de Eden e Ackermann (1998), a construção do mapa cognitivo dá-se primeiramente pela escrita de uma definição do problema pelo facilitador. A partir dela, é necessário solicitar ao decisor, ou ao grupo de decisores, que busquem identificar uma alternativa oposta a essa, considerando o contexto da problemática abordada. A soma das duas frases é o conceito, o qual é escrito de forma curta e clara. O passo seguinte consiste na condução do decisor ao desenvolvimento de ideias, na qual o facilitador deve contribuir ativamente introduzindo questões que proporcionem a identificação das ideias e as inter-relações entre elas.

Costa e Krucken (2004) apontaram que o processo de reflexão sobre o mapa construído permite ao decisor a obtenção de um conhecimento mais profundo sobre as questões que permeiam o problema. Neste sentido, o processo de construção do mapa permite a discussão acerca do problema, a elaboração de categorias e lógica para compreensão, além da elaboração de ações para "solução" da problemática em questão.

Para Jardim (2001), a compreensão do MC a partir da análise de hierarquia entre os constructos é fundamental para identificar as possíveis ligações de influência entre duas variáveis e o quanto uma pode explicar a outra. Além disso, o conhecimento dos constructos tipo cabeça (aqueles que não saem setas) e do tipo cauda (aqueles que não entram setas), no qual o primeiro aponta para os objetivos que precisam ser levados em consideração, ao passo que o segundo identifica formas de atender aos objetivos de decisor, são formas fundamentais para o entendimento do mapa cognitivo (Ensslin, Montibeller, \& Noronha, 2001).

São encontrados na literatura vários trabalhos que utilizaram o mapa cognitivo para estruturação de problemas, porém em contextos e problemas diferentes, como no segmento farmacêutico (S. de Almeida, Morais, \& de Almeida, 2014), para o planejamento estratégico situacional (Rieg \& Araújo, 2003), na gestão estratégica de resíduos sólidos (Souza \& Cordeiro, 2010), em recursos hídricos (Silva, Fontana, \& Morais 2015), entre outros. 


\subsection{Método PROMETHEE II}

Os métodos multicritério de apoio à decisão $(M C D A)$ se apresentam em três tipos principais: critério único de síntese, sobreclassificação e interativos (Roy, 1996; Vincke, 1992; Pardalos, Siskos, \& Zopounidis, 1995). A escolha do método MCDA vai depender de fatores como (Keeney \& Raiffa, 1976): problema em análise, do contexto em questão, da estrutura de preferências do decisor e da problemática envolvida.

Neste sentido, os métodos de sobreclassificação têm como principal objetivo a construção de relações binárias que representam as preferências do tomador de decisão, a qual é não compensatória (Légel \& Martel, 2002). Entre os métodos de sobreclassificação destacam-se os métodos da família PROMETHEE E ELECTRE.

Segundo Jannuzzi, Miranda e Silva (2009), o método PROMETHEE II reúne características interessantes para seu emprego nos processos decisórios típicos por pelo menos dois motivos: primeiro, por ser de fácil entendimento, potencializando a transparência do processo decisório; e segundo, porque não requer um processo interativo exaustivo, o que tornaria a aplicação de outros métodos inviável.

A família PROMETHEE se baseia em duas fases: construção da relação de sobreclassificação, a partir das informações entre as alternativas e os critérios, e a exploração dessa relação para o apoio à decisão (Brans \& Mareschal, 2002). Este método estabelece uma estrutura de preferência entre as alternativas discretas, a partir de uma função de preferência entre as alternativas em cada critério (Araújo \& de Almeida, 2009). Essa função indica a intensidade da preferência de uma alternativa em relação à outra, com o valor que varia entre 0 (indiferença) e 1 (preferência) e pode ser representada de seis formas básicas (I- critério usual, II- Quase critério, III-Critério de preferência linear, IV-Critério nível, VCritério de preferência linear com zona de indiferença, e VI- Gaussiana) em que o decisor estabelece suas preferências usando a forma mais adequada para cada critério (Brans \& Mareschal, 2002).

Na fase de exploração da relação de sobreclassificação são usados dois indicadores (A.T. de Almeida, 2013):

- Fluxo de Sobreclassificação de saída $\Phi^{+}(a)$ da alternativa "a", que representa a intensidade de preferência de "a" sobre todas as alternativas " $b$ " do conjunto de alternativas (quanto maior for este indicador, melhor a alternativa):

$$
\Phi^{+}(a)=\sum_{b \in A} \pi(a, b)
$$

- Fluxo de Sobreclassificação de entrada $\Phi^{-}$(a) da alternativa "a", que representa a intensidade de preferência de todas as alternativas " $b$ " do conjunto de alternativas sobre a alternativa "a" (quanto menor este indicador, melhor a alternativa): 


$$
\Phi^{-}(\mathrm{a})=\sum_{b \in A} \pi(b, a)
$$

Por fim, o fluxo de sobreclassificação líquido $\Phi($ a) é obtido pela relação entre os dois indicadores anteriores:

$$
\Phi(\mathrm{a})=\Phi^{+}(\mathrm{a})-\Phi^{-}(\mathrm{a})
$$

O PROMETHEE II baseia-se no fluxo líquido $\Phi$ (a) para que a partir dele as alternativas sejam ranqueadas em ordem decrescente, estabelecendo-se uma pré-ordem completa entre elas (Brans, Mareschal \& Vincke, 1986).

\section{Modelo de decisão na escolha entre produzir ou terceirizar}

O modelo de decisão proposto neste trabalho é baseado em oito etapas e possui como objetivo a priorização de alternativas que devem ser terceirizadas ou produzidas internamente. A Figura 1 representa as etapas deste modelo, o qual pode requerer a figura do facilitador para mediação.

Figura 1 - Etapas do modelo de decisão proposto

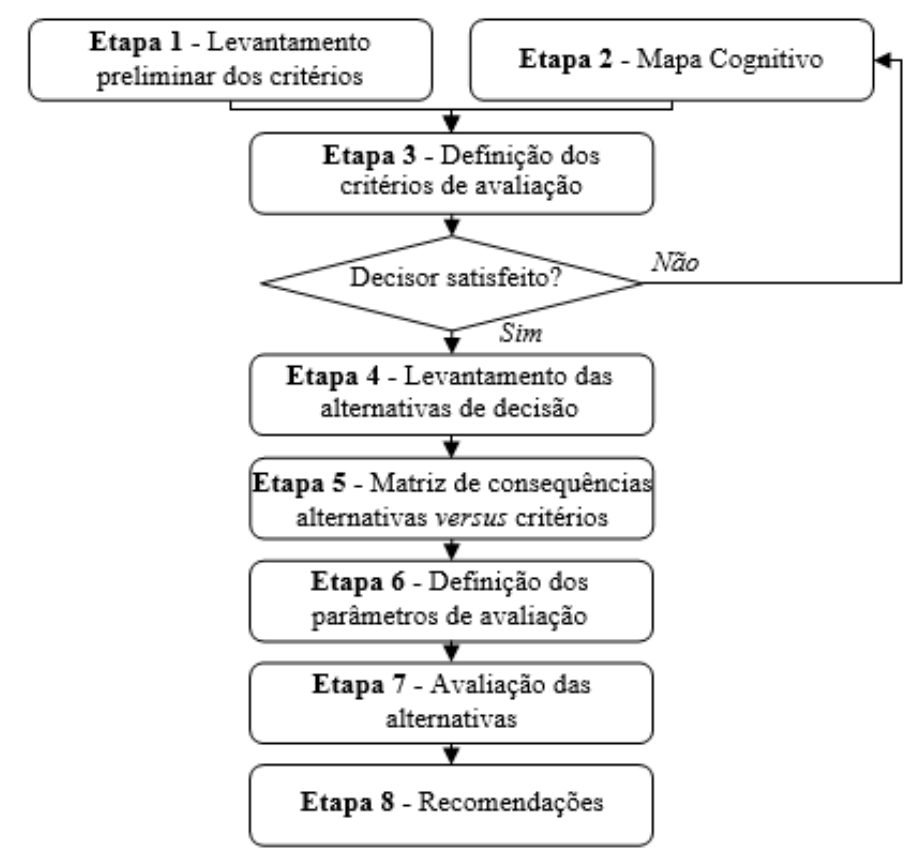

Fonte: Os autores

A seguir apresenta-se uma descrição detalhada de cada etapa necessária à aplicação do modelo proposto. 
- Etapa 1 - Levantamento preliminar dos critérios- Na primeira etapa do modelo proposto realiza-se um levantamento na literatura sobre a prática de terceirização. O objetivo desta etapa consiste em analisar e estabelecer os critérios usuais relativos à terceirização.

- Etapa 2 - Mapa Cognitivo- Nesta etapa definem-se os atores do processo decisório. Definido o decisor, são realizadas reuniões por meio de entrevistas para elaboração do Mapa Cognitivo (MC). Por fim, é importante que o analista exponha ao decisor o Mapa Cognitivo que foi construído, a fim de verificar se o decisor se sente representado no que diz respeito à sua opinião acerca do contexto. O objetivo do $M C$ é identificar os critérios relevantes para terceirização, de acordo com a perspectiva do decisor no contexto observado. Assim, pretende-se extrair do decisor critérios não observados na Etapa 1.

- Etapa 3 - Definição dos critérios de avaliação - Nesta etapa os critérios de avaliação são definidos após uma análise dos critérios observados pela literatura (Etapa 1) e o Mapa Cognitivo do decisor (Etapa 2), observando pontos de convergência e divergência. Definidos os critérios, devem-se estabelecer as escalas de avaliação de cada critério (escala verbal ou numérica), bem como os objetivos (maximizar ou minimizar).

Importante ressaltar que caso o decisor não esteja satisfeito com os critérios definidos, o mesmo pode retornar a etapa anterior para um melhor entendimento ou até mesmo modificação de pontos que se mostrarem duvidosos ou diferentes da percepção do mesmo sobre o problema. Logo, esta etapa deve ser bastante discutida, uma vez que subsidia a avaliação. Os critérios obtidos devem ser significativos e claros, de maneira que não haja interpretações errôneas por parte do decisor ou analista.

- Etapa 4 - Levantamento das alternativas de decisão - Esta etapa consiste na definição das alternativas de decisão, ou seja, as possíveis opções relacionadas à problemática de terceirização. A priori é possível que o decisor já disponha de um conjunto inicial de alternativas, tornando necessário apenas a determinação dos critérios e parâmetros para avaliação das mesmas. Entretanto, caso o decisor não disponha de um rol de alternativas, a metodologia de mapeamento cognitivo pode ser usada também para obtenção destas informações.

- Etapa 5 - Matriz de consequências alternativas versus critérios - De posse dos critérios de avaliação (Etapa 3) e alternativas de decisão (Etapa 4) é possível construir a matriz de 
consequências alternativas versus critérios. Esta matriz consiste na avaliação de cada alternativa em cada critério, por meio das escalas de avaliação definidas.

- Etapa 6 - Definição dos parâmetros de avaliação - Realizadas as etapas anteriores, estabelecem-se, nesta etapa, as preferências do decisor, ou seja, os parâmetros do método escolhido. No modelo de decisão proposto, o método multicritério utilizado foi o método PROMETHEE II. A escolha desse método foi baseada nas seguintes características: (i) Necessidade de ordenar as alternativas de decisão; e (ii) Racionalidade não compensatória, por acreditar que ao definir se uma atividade será ou não realizada internamente, se procura observar o desempenho global desta alternativa. Neste caso não seria interessante que um desempenho péssimo em um critério fosse compensado por um ótimo desempenho em outro critério. Como exemplo, pode-se supor um cliente que não aceita receber um produto rapidamente se para isso perder qualidade. Desta forma, os parâmetros que devem ser estabelecidos são: pesos dos critérios, limiares de preferência, indiferença e de veto. Importante destacar que estes podem mudar de acordo com as preferências do decisor.

- Etapa 7-Avaliação das alternativas- De posse de todas as informações aplica-se o método multicritério PROMETHEE II. Para isto, pode-se usar o software Visual PROMETHEE, desenvolvido por Bertrand Mareschal. Nesta etapa é realizada, também, uma análise de sensibilidade para avaliar a estabilidade e robustez do modelo.

- Etapa 8 - Recomendações - Por fim, são fornecidas recomendações ao decisor. Ressaltase que apesar da solução refletir as preferências do decisor, o mesmo ainda é soberano em adotar ou não a solução recomendada.

\section{Estudo de caso}

O estudo de caso foi realizado em uma empresa de confecção de pequeno porte do interior de Pernambuco, a qual atua no segmento de moda e praia, com um portfólio de produtos enxuto: linha feminina, linha masculina e uma linha infantil. Localizada na cidade de Caruaru, a empresa atua no mercado há cerca de 7 anos. O quadro funcional é composto por 12 funcionários, sendo 03 vinculados à área administrativa e os demais à produção.

Avaliando o aumento da demanda e considerando a necessidade salutar de manter custos em níveis baixos e qualidade em um padrão que atenda as exigências de mercado, a empresa vem buscando 
alternativas mais consistentes que possam atender aos seus objetivos e mantê-la a frente da concorrência. Desta forma, as etapas do modelo proposto aplicadas na empresa objeto de estudo são apresentadas e discutidas na sequência.

\subsection{Etapa 1 - Levantamento preliminar dos critérios}

A análise da literatura deu-se a partir de periódicos de relevância na área, disponibilizados nas principais bases científicas, como Scielo e Science Direct. Ao examinar os critérios (estes incluem tanto medidas quantitativas e qualitativas), podem-se evitar decisões errôneas, baseadas muitas vezes na intuição, como a de que o custo por si só é um fator decisivo à terceirização. O Quadro 1 apresenta os critérios observados na análise da literatura.

Quadro 1 - Conjunto preliminar dos critérios

\begin{tabular}{|c|c|c|}
\hline Critérios & Descrição & Autores \\
\hline Custos & $\begin{array}{l}\text { Manter custos de produção mais } \\
\text { baixos possíveis. Ao se passar para } \\
\text { terceiros, os custos fixos são } \\
\text { transformados em variáveis. }\end{array}$ & $\begin{array}{l}\text { Fill e Visser (2000); Mclvor (2000); A. T. de } \\
\text { Almeida (2007); Araz, Ozfirat e Ozkarahan } \\
\text { (2007); Wang e Yang (2007); Kahraman, } \\
\text { Engin, Kabak e Kaya, (2009); Valois e de } \\
\text { Almeida (2009); Chen, Wang e Wu (2011); } \\
\text { Kaya (2012); Qureshi, Salman e Khalid } \\
\text { (2013); Kahraman, Öztaysi e Onar (2016); } \\
\text { Gürcan, Yazici, Beyca, Arslan e Eldemir } \\
\text { (2016); Tavana, Zareinejad, Di Caprio e } \\
\text { Kaviani (2016) }\end{array}$ \\
\hline Qualidade & $\begin{array}{l}\text { Realização do produto ou serviço } \\
\text { de acordo com as exigências } \\
\text { estabelecidas. A qualidade é válida } \\
\text { tanto para os clientes internos da } \\
\text { cadeia de suprimentos quanto para } \\
\text { o consumidor final. }\end{array}$ & $\begin{array}{l}\text { Araz, Ozfirat e Ozkarahan (2007); Wang e } \\
\text { Yang (2007); Valois e de Almeida (2009); } \\
\text { Chen, Wang e Wu (2011); Kaya (2012); } \\
\text { Qureshi, Salman e Khalid (2013); Morais, } \\
\text { Costa e de Almeida (2014); Kahraman, } \\
\text { Öztaysi e Onar (2016) }\end{array}$ \\
\hline Credibilidade & $\begin{array}{l}\text { Garantir a cooperação entre as } \\
\text { partes envolvidas, o que inclui o } \\
\text { compartilhamento de riscos. }\end{array}$ & $\begin{array}{l}\text { Olson (2006); Araz, Ozfirat e Ozkarahan } \\
\text { (2007); Kahraman, Engin, Kabak e Kaya, } \\
\text { (2009); Kahraman, Öztaysi e Onar (2016) }\end{array}$ \\
\hline Flexibilidade & $\begin{array}{l}\text { Capacidade de mudar o que se faz, } \\
\text { ou seja, de se adaptar o seu } \\
\text { sistema de acordo com as } \\
\text { necessidades do contratante. }\end{array}$ & $\begin{array}{l}\text { Araz, Ozfirat e Ozkarahan (2007); Wang e } \\
\text { Yang (2007); Valois e de Almeida (2009); } \\
\text { Chen, Wang e Wu (2011); Kahraman, } \\
\text { Öztaysi e Onar (2016) }\end{array}$ \\
\hline Velocidade & $\begin{array}{l}\text { Rapidez com o que a atividade ou } \\
\text { serviço é realizado. Representa } \\
\text { também o desempenho no tempo } \\
\text { de entrega dos terceirizados. }\end{array}$ & $\begin{array}{l}\text { A. T. de Almeida (2007); Araz, Ozfirat e } \\
\text { Ozkarahan (2007); Kahraman, Engin, } \\
\text { Kabak e Kaya, (2009); Valois e de Almeida } \\
\text { (2009) }\end{array}$ \\
\hline
\end{tabular}

Fonte: Os autores

Tais critérios apresentados mostram as diversas discussões existentes na literatura sobre o tema. De acordo com o Quadro 1, observa-se que os critérios custo e qualidade são os mais relatados pela literatura para a decisão de terceirização, seguidos dos critérios flexibilidade, credibilidade e velocidade. 
Redução de custos administrativos e de pessoal e economia de escala são benefícios esperados com a adoção da terceirização. Além disso, a terceirização possibilita a transferência de atividades ou serviços para empresas especializadas. Sendo assim, além de oferecer um produto ou serviço com a qualidade esperada, a empresa que terceiriza consegue focar em seu core competence. Entretanto, destaca-se que os critérios retratados no Quadro 1 podem não ser considerados em todos os cenários em que se discute terceirizar ou não uma atividade ou processo. Assim, torna-se fundamental também compreender o cenário de decisão para primar os critérios que de fato são relevantes. Portanto, seguese para a Etapa 2 do modelo.

\subsection{Etapa 2 - Mapa Cognitivo}

A partir da entrevista com o decisor da empresa estudada, foi elaborado um mapa cognitivo, em que teve com o rótulo: "Quais fatores devem ser considerados na decisão entre terceirizar ou produzir?". O mapa cognitivo resultante pode ser visualizado na Figura 2.

Figura 2 - Mapa Cognitivo

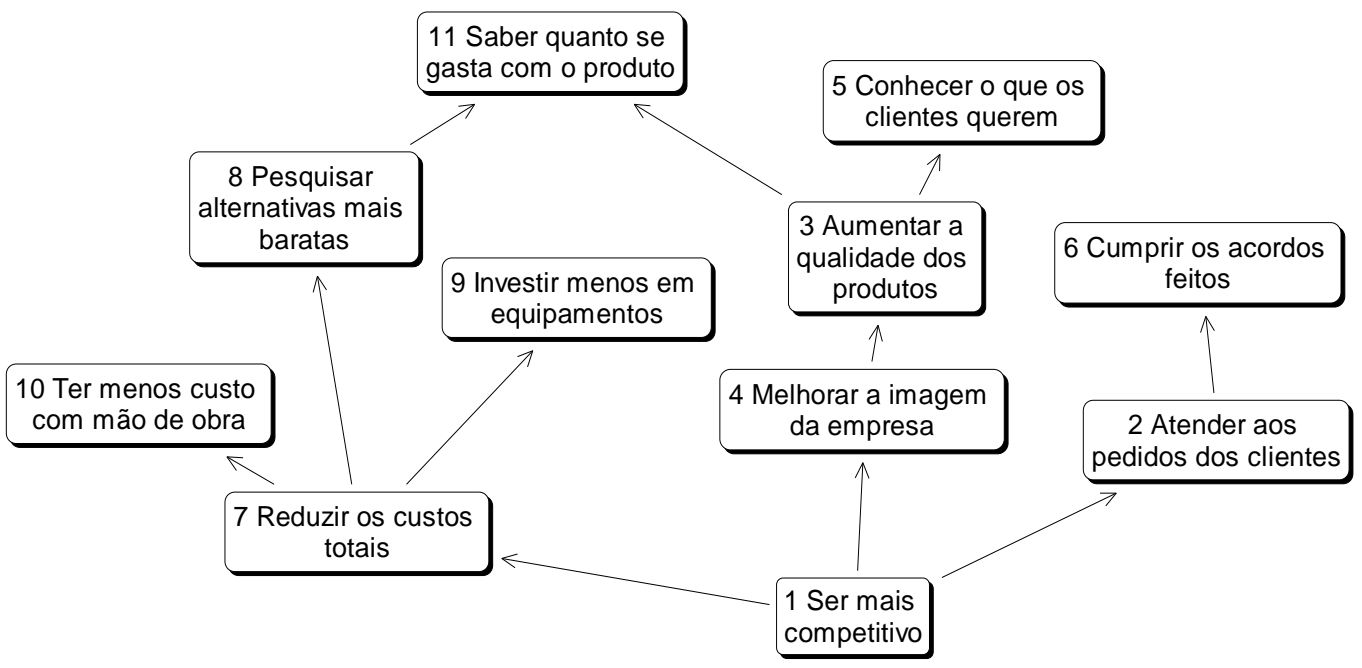

Fonte: Os autores

Foi possível identificar no mapa cognitivo uma preocupação em se tornar mais competitivo no mercado de atuação, evidenciado pelos elementos primários de avaliação: (1) reduzir os custos totais, (2) melhorar a imagem da empresa e (3) atender aos pedidos dos clientes. Por meio destes elementos, foram construídos conceitos na proposta do modelo bipolar que demonstram possíveis ligações entre eles e estratégias de ação. E, por fim, com base em tudo que foi exposto, a hierarquização dos conceitos. 
Partindo para uma análise dos constructos identificados, foi possível identificar cinco constructos tipo cabeça: conhecer o que os clientes querem (constructo 5), cumprir os acordo feitos (constructo 6), investir menos em equipamentos (constructo 9), ter menos custo com mão de obra (constructo 10) e saber quanto se gasta com o produto (constructo 11). Tais constructos são considerados objetivos ou consequências dos demais constructos abaixo deles.

Como opções estratégicas, ou seja, as alternativas possíveis para as cabeças, identificaram-se quatro constructos: atender aos pedidos dos clientes (constructo 2), aumentar a qualidade dos produtos (constructo 3), reduzir os custos totais (constructo 7) e pesquisar alternativas mais baratas (constructo 8).

Por sua vez, foi detectado apenas um constructo tipo cauda: ser mais competitivo (constructo 1). Este, conforme a literatura é considerado a causa primária, ponto fundamental a ser atingido ou situação desencadeadora do problema. Neste sentido, para este decisor a causa primária do problema de decisão é garantir uma maior competitividade.

Em relação ao constructo implosão, o qual consiste no constructo que está sendo afetado por vários outros, detectou-se o constructo "saber quanto se gasta com o produto" (constructo 11), como ponto de convergência. Relativo ao constructo explosão, que por sua vez representa constructo causa, pois afeta vários outros, tem-se os constructos ser mais competitivo (constructo 1) e reduzir os custos totais (constructo 7), tratando-se de pontos de divergência. Entretanto, ressalta-se que a centralidade cognitiva do decisor, ou seja, o constructo dominante, é reduzir os custos totais (constructo 7).

Após a realização e análise do mapa cognitivo do decisor e de posse do que foi observado pela literatura, torna-se possível a definição dos critérios de avaliação.

\subsection{Etapa 3 - Definição dos critérios de avaliação}

A partir da comparação entre o que foi observado na literatura e o mapa cognitivo do decisor, foi possível identificar alguns pontos convergentes em relação aos critérios considerados na decisão analisada. Primeiro, que houve uma preocupação com o custo, o qual se desdobra em custos com mão de obra e equipamentos, e sustenta a ideia de se buscar alternativas mais baratas e redutoras, como a opção de terceirizar. Segundo, ficou evidente, também, a necessidade de pelo menos manter a qualidade dos produtos da empresa, em um cenário menos otimista. Outro ponto importante detectado foi o de conseguir cumprir os acordos estabelecidos e atender aos pedidos dos clientes, o que requer credibilidade e flexibilidade como critérios extremamente críticos na decisão entre terceirizar e produzir. 
Apesar dos pontos de convergência, foi possível observar algumas divergências. Pelo o que foi retratado pelo decisor, a sua preocupação com a qualidade refere-se apenas à satisfação dos clientes por meio da qualidade do produto, não observando o processo. Além disso, outra divergência observada diz respeito ao critério velocidade que foi retratado pela literatura (ver Quadro 1), porém não destacado como critério de avaliação pelo decisor. Neste sentido, o decisor avaliou e preferiu o termo responsividade, dando ênfase à agilidade conforme as mudanças do mercado e necessidades dos clientes, e não apenas ao processo de produção atual, ou seja, sua preocupação foco é o atendimento das necessidades do cliente, ou nível de serviço oferecido.

Assim, mediante os critérios elencados pela literatura e pelo mapeamento cognitivo do decisor, sobre os critérios relevantes na decisão entre terceirizar e produzir voltado ao setor confecção de Pernambuco, foram estabelecidos os critérios, os quais podem ser visualizados no Quadro 2. Tais critérios foram avaliados por julgamentos subjetivos do decisor mediante a utilização de uma escala nominal-ordinal sugerida pelo mesmo (Muito alta, Alta, Razoável, Baixa, Muito baixa).

Quadro 2 - Critérios de avaliação

\begin{tabular}{|l|l|}
\hline \multicolumn{1}{|c|}{ Critério } & \multicolumn{1}{c|}{ Descrição } \\
\hline C1 - Custo total & Valor estimado da adoção da alternativa \\
\hline C2 - Qualidade & Atendimento as exigências solicitadas pelos clientes sem erros \\
\hline C3 - Credibilidade & Manutenção do funcionamento esperado independente das circunstâncias \\
\hline C4 - Flexibilidade & Atendimento a demanda em suas mais variadas características \\
\hline C5 - Responsividade & Capacidade de resposta aos clientes de forma precisa e rápida \\
\hline
\end{tabular}

Fonte: Os autores

Conforme a natureza do problema buscou-se por terceirizar alternativas que fossem mais dispendiosas em termos de custo de produção interna e que oferecessem riscos consideráveis nos demais critérios aqui estabelecidos. Em outras palavras, quanto menor for o valor atribuído ao critério Custo Total e maiores forem os valores atribuídos aos demais critérios (Qualidade, Credibilidade, Flexibilidade e Responsividade) haverá uma maior tendência do decisor em terceirizar essa atividade. Sendo assim, buscou-se por minimizar C1 e maximizar C2, C3, C4 e C5.

\subsection{Etapa 4 - Levantamento das alternativas de decisão}

A partir da proposição do decisor sobre terceirização, considerando como alvo o setor de costura, foi obtido um conjunto com oito alternativas, as quais são descritas no Quadro 3. 
Quadro 3 - Alternativas levantadas

\begin{tabular}{|l|l|}
\hline \multicolumn{1}{|c|}{ Alternativas } & \multicolumn{1}{c|}{ Descrição } \\
\hline A1 - Não terceirizar & Realizar toda a produção internamente. \\
\hline $\begin{array}{l}\text { A2 - Terceirizar apenas a linha } \\
\text { infantil }\end{array}$ & $\begin{array}{l}\text { Terceirizar unicamente a linha infantil, responsável por cerca de 20\% da } \\
\text { produção no setor de costura. }\end{array}$ \\
\hline $\begin{array}{l}\text { A3 - Terceirizar apenas a linha } \\
\text { feminina }\end{array}$ & $\begin{array}{l}\text { Terceirizar unicamente a linha feminina, responsável por cerca de 50\% da } \\
\text { produção no setor de costura. }\end{array}$ \\
\hline $\begin{array}{l}\text { A4 - Terceirizar apenas a linha } \\
\text { masculina }\end{array}$ & $\begin{array}{l}\text { Terceirizar unicamente a linha masculina, responsável por cerca de 30\% da } \\
\text { produção no setor de costura. }\end{array}$ \\
\hline $\begin{array}{l}\text { A5 - Terceirizar as linhas } \\
\text { infantil e feminina }\end{array}$ & $\begin{array}{l}\text { Terceirizar as linhas infantil e feminina, responsáveis por cerca de 70\% da } \\
\text { produção no setor de costura. }\end{array}$ \\
\hline $\begin{array}{l}\text { A6 - Terceirizar as linhas } \\
\text { infantil e masculina }\end{array}$ & $\begin{array}{l}\text { Terceirizar as linhas infantil e masculina, responsáveis por cerca de 50\% da } \\
\text { produção no setor de costura. }\end{array}$ \\
\hline $\begin{array}{l}\text { A7 - Terceirizara as linhas } \\
\text { feminina e masculina }\end{array}$ & $\begin{array}{l}\text { Terceirizar as linhas feminina e masculina, responsáveis por cerca de 80\% } \\
\text { da produção no setor de costura. }\end{array}$ \\
\hline A8 - Terceirizar totalmente & Terceirizar 100\% a produção no setor de costura. \\
\hline
\end{tabular}

Fonte: Os autores

Levantados os critérios desejados e as alternativas a serem avaliadas, seguiu-se à construção da matriz de consequências.

\subsection{Etapa 5 - Matriz de consequências alternativas versus critérios}

A partir das etapas anteriores, bem como do cenário proposto e utilizando uma avaliação intracritério, com julgamentos subjetivos realizados pelo decisor de acordo com a escala ordinal estabelecida na Etapa 3, obteve-se a matriz de consequências apresentada no Quadro 4.

Quadro 4-Matriz de consequências

\begin{tabular}{|c|c|c|c|c|c|}
\cline { 2 - 5 } \multicolumn{1}{c|}{} & C1 & C2 & C3 & C4 & C5 \\
\hline A1 & Muito alta & Muito alta & Baixa & Muito baixa & Muito baixa \\
\hline A2 & Razoável & Razoável & Muito baixa & Muito baixa & Baixa \\
\hline A3 & Baixa & Alta & Razoável & Razoável & Alta \\
\hline A4 & Razoável & Alta & Razoável & Razoável & Razoável \\
\hline A5 & Baixa & Razoável & Razoável & Razoável & Alta \\
\hline A6 & Razoável & Razoável & Razoável & Razoável & Alta \\
\hline A7 & Razoável & Razoável & Alta & Alta & Alta \\
\hline A8 & Muito baixa & Alta & Alta & Alta & Alta \\
\hline Objetivo & Min & Max & Max & Max & Max \\
\hline
\end{tabular}

Fonte: Os autores

Estabelecida a matriz de consequências alternativas versus critérios, segue-se para a definição dos parâmetros de avaliação. 


\subsection{Etapa 6 - Definição dos parâmetros de avaliação}

O decisor considerou a função de preferência 'critério usual' para todos os critérios. Assim, não foram definidos limiares de preferência ou indiferença. O limiar de veto também foi desconsiderado pelo mesmo, restando apenas o estabelecimento de pesos aos critérios, que foram: $C 1=0,3 ; C 2=0,2$; $C 3=0,15 ; C 4=0,15$ e $C 5=0,2$.

Vale ressaltar que o decisor em questão não apresentou quaisquer dificuldades em definir os parâmetros de avaliação.

\subsection{Etapa 7 - Avaliação das alternativas}

Portanto, realizadas as etapas anteriores obteve-se o resultado do método PROMETHEE II, com o auxílio do software Visual PROMETHEE, conforme mostrado na Tabela 1.

Tabela 1 - Ranque das alternativas pelo PROMETHEE II

\begin{tabular}{ccc}
\hline $\begin{array}{c}\text { Posição no } \\
\text { Ranque }\end{array}$ & Alternativa & Fluxo Líquido $(\Phi)$ \\
\hline 1 & A8 & 0,7286 \\
2 & A3 & 0,3429 \\
3 & A7 & 0,1429 \\
4 & A5 & 0,1429 \\
5 & A4 & $-0,0857$ \\
6 & A6 & $-0,1143$ \\
7 & A1 & $-0,5357$ \\
8 & A2 & $-0,6214$ \\
\hline
\end{tabular}

Fonte: Os autores

Realizando uma análise de sensibilidade a partir da variação do peso dos critérios em $\pm 10 \%$, redistribuindo os pesos aos demais critérios igualmente, observou-se que a ordenação obtida pelo PROMETHEE II não apresentou variações, o que reflete a robustez do método.

\subsection{Etapa 8 - Recomendações}

Mediante a análise dos resultados obtidos, tem-se que a alternativa A8 (Terceirizar totalmente) apresenta o maior fluxo líquido, seguida das alternativas A3 (Terceirizar apenas a linha feminina) e A7 (Terceirizar a linha feminina e a masculina). Portanto, recomendou-se ao decisor a adoção da alternativa A8, ou seja, terceirizar $100 \%$ a produção do setor de costura. 


\section{Discussões}

A metodologia proposta por este trabalho permitiu melhor compreensão do problema a partir da revisão da literatura e mediante o uso de mapa cognitivo para definição dos fatores importantes considerados na decisão de terceirização.

Observou-se que a literatura apresenta um conjunto de critérios frequentemente utilizados para este tipo de decisão. Entretanto, considerar esses critérios sem nenhuma avaliação das particularidades do cenário observado pode levar a decisões errôneas, bem como o contrário é verdadeiro. Tal fato ficou evidente quando se observou a percepção do decisor a respeito do critério qualidade em relação ao produto e responsividade em relação ao nível de serviço oferecido, não considerando a qualidade e velocidade do processo propriamente dito. Isto reforça a necessidade do uso de metodologias que auxiliem os decisores na compreensão do seu conjunto de critérios de avaliação.

Outro fator importante e que merece destaque diz respeito à utilização do método multicritério PROMETHEE II. Ele mostrou-se satisfatório, dado que foi possível propor uma recomendação final cabível, a partir da ordenação entre as alternativas, atendendo aos critérios propostos.

No que concerne a essa recomendação apresentada ao decisor, ela mostrou-se coerente com o setor de confecções quando se toma decisões entre terceirizar ou produzir, dado que menores custos e maior economia de escala podem ser obtidos a partir de uma produção maior. No caso da terceirização de $100 \%$ da produção, o decisor pode direcionar seus esforços para manutenção do seu core competence, isto é, desenvolver novos métodos de penetração de mercado, melhorar seus processos internos, aumentar/diversificar seu mix de produtos, entre outros, promovendo seu diferencial competitivo no mercado.

Outra questão interessante para discussão é a relação entre terceirizar apenas a linha infantil (A2) ou não terceirizar (A1), na qual a alternativa $\mathrm{A} 1$ apresenta um fluxo líquido maior, isto é, terceirizar um percentual mínimo da costura não é uma alternativa atraente para o decisor em questão. Neste ponto, o impacto nos custos totais bem como a falta de priorização da empresa terceirizada associado ao baixo volume são fatores que tornam tais alternativas menos interessantes.

Ressalta-se ainda que o decisor mostrou-se receptivo com a solução do modelo proposto, sentindo-se confiante em adotar a solução recomendada.

\section{Conclusão}

A partir do levantamento realizado na literatura, identificaram-se os critérios fundamentais a serem avaliados na tomada de decisão de terceirização. Porém, com a construção de um mapa cognitivo, que retratou a perspectiva de um decisor do APL de confecções do Agreste Pernambucano, 
foi possível direcionar melhor os critérios conforme foco desejado pelo decisor. A utilização de tal ferramenta no contexto de decisão apresentado proporcionou uma ampliação da visão do decisor, apresentando detalhamento importante para a tomada de decisão.

Do ponto de vista prático, a partir da aplicação do modelo proposto, obteve-se como recomendação a priorização da alternativa de terceirização total. Isto se deve, sobretudo, a fatores realísticos do segmento de confecção, como economias de escala, custos totais de produção e responsividade das empresas terceirizadas. Neste sentido, a adoção da alternativa A8 (Terceirizar totalmente) possibilita que a empresa se dedique a desenvolver seu core competence, fundamentando suas ações e direcionando-as para obter vantagens competitivas a frente dos seus concorrentes.

Não obstante, o modelo mostrou-se capaz de absorver as necessidades e fatores fundamentais do decisor no ato da escolha, tornando clara a preocupação emergente com a satisfação do cliente ao mesmo tempo em que há um olhar diferenciado sobre os custos envolvidos no processo, pontos estes que não poderiam ser avaliados de forma compensatória.

Já do ponto de vista teórico, o modelo de decisão proposto auxilia na definição dos critérios de avaliação, apoiando a tomada decisão de forma eficaz. Além de uma recomendação de solução, o modelo proposto aborda o problema de decisão entre terceirizar ou produzir de forma compreensível, permitindo ao decisor maior racionalidade em sua decisão. Vale ressaltar que este modelo pode ser estendido para outras áreas de negócios que se encaixem neste cenário.

Ademais, apesar da robustez demonstrada pelo modelo e considerando os resultados apresentados, sugere-se a análise e identificação de possíveis restrições que possam fazer parte deste contexto de decisão, sejam estas temporais, físicas ou financeiras.

\section{Agradecimentos}

Os autores agradecem a Coordenação de Aperfeiçoamento de Pessoal de Nível Superior (CAPES) e a Fundação de Amparo a Ciência e Tecnologia do Estado de Pernambuco (FACEPE).

\section{Referências}

Araújo, A. G. de; \& De Almeida, A. T. (2009). Apoio à decisão na seleção de investimentos em petróleo e gás: uma aplicação utilizando o método PROMETHEE. Gestão \& Produção, 16(4), 534-543.

Araz, C.; Ozfirat, P. M.; \& Ozkarahan, I. (2007). An integrated multicriteria decision-making methodology for outsourcing management. Computers \& Operations Research, 34, 3738-3756. 
Associação Brasileira de Indústria Têxtil e de Confecção. (2013). Disponível em: < http://www.abit.org.br/conteudo/links/publicacoes/cartilha_rtcc.pdf>. Acesso em: 29 de setembro de 2017.

Associação Brasileira de Indústria Têxtil e de Confecção. (2015). Disponível em: < http://www.abit.org.br/dadosdosetor/>. Acesso em: 19 de setembro de 2017.

Brans, J. P.; \& Mareschal, B. (2002). PROMÉTHÉE-GAIA: une méthodologie d'aide à la décision em présence de critères multiples. Bruxelles: Éditions de L'Université de Bruxelles.

Brans, J. P; Mareschal, B; \& Vincke, P. (1986). A preference ranking organization method - the PROMETHEE method for multiple criteria decision-making. Management Science, 31, 647- 656.

Cachon, G. P.; \& Harker, P. T. (2002). Competition and outsourcing with scale economies. Management Science, 48, 1314-1333.

Cervi, A. F. C.; \& Carpinetti, L. C. R. (2017). Competências essenciais da organização: avaliação da terceirização baseada no método ELECTRE TRI. Pesquisa Operacional para o Desenvolvimento, 9(2), $95-108$

Chen, Y. H.; Wang, T. C.; \& Wu, C. Y. (2011). Strategic decisions using the fuzzy PROMETHEE for IS outsourcing. Expert Systems with Applications, 38, 13216-13222.

Costa, M. D.; \& Krucken, L. (2004). Aplicações de mapeamento do conhecimento para a competitividade empresarial. In: KM BRASIL 2004 - Gestão do Conhecimento na Política Industrial Brasileira, São Paulo, Brasil.

Departamento Intersindical de Estatística e Estudos Socioeconômicos. (2012). Terceirização e negociação coletiva: velhos e novos desafios para o movimento sindical brasileiro. Nota técnica, 112. Disponível em: <https://www.dieese.org.br/notatecnica/2012/notaTec112terceirizacao.pdf> Acesso em: 23 de dezembro de 2017.

De Almeida, A. T. (2007). Multicriteria decision model for outsourcing contracts selection based on utility function and ELECTRE method. Computers \& Operations Research, 34, 3569-3574.

De Almeida, A.T. (2013). Processo de decisão nas organizações: construindo modelos de decisão multicritério. São Paulo: Atlas.

De Almeida, S.; Morais, D. C.; \& De Almeida, A. T. (2014). Agregação de pontos de vista de stakeholders utilizando o Value Focused Thinking associado à mapeamento cognitivo. Production, 24(1), 144-159.

Eden, C. (2004). Analyzing cognitive maps to help structure issues or problems. European Journal of Operational Research, 159(3), 673-686.

Eden, C.; \& Ackermann, F. (1998). Making Strategy: The Journey of Strategy Management. Sage, London.

Ensslin, L.; Giffhorn, E.; Ensslin, S. R.; \& Vianna, W. B. (2010). Avaliação do desempenho de empresas terceirizadas com o uso da metodologia multicritério de apoio à decisão-construtivista. Pesquisa Operacional, 30(1), 125-152. 
Ensslin, L.; \& Montibeller, G., Neto. (1998). Mapas cognitivos no apoio à decisão. In: XVIII Encontro Nacional de Engenharia de Produção, Rio de Janeiro, Anais.

Ensslin, L.; Montibeller, G., Neto; \& Noronha, S. M. (2001). Apoio à decisão: metodologias para estruturação de problemas e avaliação multicritério de alternativas. Florianópolis: Insular.

Fill, C.; \& Visser, E. (2000). The outsourcing dilemma: a composite approach to the make or buy decision. Management Decision, 38(1), 43-50.

Freitas, E. C.; Schlindwein, J.; \& Barth, M. (2010). A gestão de processos terceirizados como fator de qualidade em micro e pequenas empresas de prestação de serviços. Revista eletrônica de Gestão de Negócios, 6(2), 41-71.

Gunasekaran, A.; Irani, Z.; Choy, K.L.; Filippi, L.; \& Papadopoulos, T. (2015). Performance measures and metrics in outsourcing decisions: a review for research and applications. International Journal of Production Economics, 161, 153-166.

Gürcan, Ö. F.; Yazici, I.; Beyca, Ö. F.; Arslan, Ç. Y.; \& Eldemir, F. (2016). Third Party Logistics (3PL) provider selection with AHP application. In: Procedia - Social and Behavioral Sciences, 235, 226234.

Jannuzzi, P. de M.; Miranda, W.L. de; \& Silva, D.S.G da. (2009). Análise multicritério e tomada de decisão em políticas públicas: aspectos metodológicos, aplicativo operacional e aplicações. Revista Informática Pública, 11(1), 69-87.

Jardim, S. B. (2001). Mapas Cognitivos: um caminho para construir estratégias. Revista Acadêmica da Face Pucrs, 12, 89-115.

Kahraman, C.; Öztaysi, B.; \& Onar, S. C. (2016). Intuitionistic fuzzy multicriteria evaluation of outsource manufacturers. IFAC-PapersOnLine, 49(12), 1844-1849.

Kahraman, C.; Engin, O.; Kabak, Ö.; \& Kaya, I. (2009). Information systems outsourcing decisions using a group decision-making approach. Engineering Applications of Artificial Intelligence, 22, 832-841.

Kaya, I. (2012). Evaluation of outsourcing alternatives under fuzzy environment for waste management. Resources, Conservation and Recycling, 60, 107-118.

Keeney, R.L.; \& Raiffa, H. (1976). Decision with Multiple Objectives: Preferences and Value Trade-offs. John Wiley \& Sons.

Kotabe, M., \& Zhao, H. (2002). A taxonomy of sourcing strategic types for MNCs operating in China. Asia Pacific Journal of Management, 19, 11-27.

Légel, J.; \& Martel, J.M. (2002). A multicriteria assignment procedure for a nominal sorting problematic. European Journal of Operational Research, 138(2), 349-364.

Lonsdale, C. (1999). Effectively managing vertical supply relationships: a risk management model for outsourcing. Supply Chain Management: an international journal, 4(4), 176-183.

Mareschal, B. (Versão 1.4). [software]. Visual PROMETHEE. Disponível em: < http://www.prometheegaia.net/software.html>. Acesso em: 30 de setembro de 2017. 
Mclvor, R. (2000) A pratical framework for understanding the outsourcing process. Supply Chain Management: an international journal, 5(1), 22-36.

Minaei, N. (2014). Do modes of transportation and GPS affect cognitive maps of Londoners? Transportation research part A: policy and practice, 70, 162-180, 2014.

Morais, D. C.; Costa, A. P. C. S.; \& De Almeida, A. T. (2014). Group decision model for outsourcing IT services. In: Procedia Technology, 16, 562-568.

Murthy, D. N. P.; Karim, M. R.; \& Ahmadi, A. (2015). Data management in maintenance outsourcing. Reliability Engineering and System Safety, 142, 100-110.

Oliveira, G. C., Neto; Contador, J. C.; Shibao, F. Y.; \& Vendrametto, O. (2013). Método para hierarquizar os critérios e indicadores de desempenho para contratação de operador logístico: estudo de caso no ramo metalúrgico. Exacta, 11(3), 259-283.

Olson, D.L. (2006). Evaluation of ERP outsourcing. Computers \& Operations Research, 34, 3715-3724.

Pardalos, P. M.; Siskos, Y.; \& Zopounidis, C. (1995). Advances in multicriteria analysis, Kluwer Academic Publishers.

Peng, J. (2012). Selection of logistics outsourcing service suppliers based on AHP. In: Energy Procedia, $17,595-601$.

Pereira, R. A.; Scur, G.; \& Consoni, F. L. (2017). Outsourcing nas atividades de desenvolvimento de novos produtos: o caso da Volkswagen do Brasil. Exacta, 15(2), 289-303.

Piccinini, V. C.; Oliveira, S. R. de; \& Fontoura, D. dos S. (2006). Setor têxtil-vestuário do Rio Grande do Sul: impactos da inovação e da flexibilização do trabalho. Ensaios FEE, 27(2), 355-376.

Prahalad, C. K.; \& Hamel, G. (1990). The core competence of the corporation. Harvard Business Review, 3-15.

Qureshi, M. A.; Salman, M.; \& Khalid, R. (2013). Development of a framework for strategic outsourcing in developing countries. International Journal of Materials, Mechanics and Manufacturing, 1(1), 9296.

Rieg, D. L.; \& Araújo, T., Filho. (2003). Mapas cognitivos como ferramenta de estruturação e resolução de problemas: o caso da pró-reitoria de extensão da UFSCar. Revista Gestão \& Produção, 10(2), 145-162.

Roy, B. (1996). Multcriteria methodology for decision aiding. Kluwer Academic Publishers.

Silva, J. L., Filho; Fontana, M. E.; \& Morais, D. C. (2015). Analyzing conflicts between decision-makers in determining criteria to evaluate segmentation in water distribution networks. In: 2015 IEEE International Conference on Systems, Man, and Cybernetics (SMC), 252-257.

Souza, R. G. de; \& Cordeiro, J. S. (2010). Mapeamento cognitivo e Balanced Scorecard na gestão estratégica de resíduos sólidos urbanos. Gestão \& Produção, 17(3), 483-496.

Tavana, M.; Zareinejad, M.; Di Caprio, D.; \& Kaviani, M. A. (2016). An integrated intuitionistic fuzzy AHP and SWOT method for outsourcing reverse logistics. Applied Soft Computing, 40, 544-557. 
Valença, M. C. de A.; \& Barbosa, A. C. Q. (2002) A Terceirização e seus impactos: um estudo em grandes organizações de Minas Gerais. Revista de Administração Contemporânea, 6(1), 163-185.

Valois, U.; \& De Almeida, A. T. (2009). Modelo de apoio à decisão multicritério para terceirização de atividades produtivas baseado no método SMARTS. Produção, 19(2), 249-260.

Vincke, P. (1992). Multicriteria decision-aid. Bruxelles: Jonh Wiley \& Sons.

Wang, J.J; \& Yang, D.L. (2007). Using a hybrid multi-criteria decision aid method for information systems outsourcing. Computers \& Operations Research, 34, 3691-3700.

Xiao, T.; Xia, Y.; \& Zhang, G.P. (2007). Strategic outsourcing decisions for manufacturers that produce partially substitutable products in a quantity-setting duopoly situation. Decision Sciences, 38, 81106. apoio à decisão entre produzir ou terceirizar. Exacta, 18(1), 53-73. https://doi.org/10.5585/Exacta.v18n1.7980 